[Aus dem hygienischen Institut der Kgl. Universität Berlin.]

(Direktor: Prof. Dr. Flügge.)

\title{
Die Gewinnung von keimfreiem Trinkwasser im Felde.
}

Von

Stabsarzt Dr. Kunow,

kommandiert zum Institut.

Die Versuche, jedem einzelnen Soldaten im Felde Mittel an die Hand zu geben, um sich aus dem zur Verfügung stehenden Oberflächenwasser in kurzer Zeit selbst ein einwandfreies und schmackhaftes Trinkwasser herzustellen, haben bisher noch nicht zu einem Verfahren geführt, welches unter Berücksichtigung des tatsächlichen Bedürfnisses der Truppen und der feldmäBigen Anwendbarkeit zu allgemeiner Einführung empfohlen werden kann. Aus rein technischen Gesichtspunkten ist man daher auch in der Armee zur Sammelversorgung übergegangen, welche der verschiedenen Größe der einzelnen taltischen Verbände angepaßt ist und die Vernichtung der Krankheitskeime durch Ablochen des Wassers erreicht. Kleine Truppenverbände bleiben jedoch bei getrennter taktischer Verwendung in der Regel auf den Inhalt der an der Zentralstelle gefüllten Feldflaschen angewiesen, da trotz der groBen Zahl der zur Entkeimung von Oberflächenwasser empfohlenen chemischen Mittel und der verschiedenen Filterapparate bisher kein Verfahren einer strengen bakteriologischen Kontrolle standgehalten hat. Trotzdem darf aber, wie Hetsch in dem Lehrbuch der Militärhygiene ausführt, „die weitere Erprobung von Filterapparaten und chemischen Mitteln für die Zwecke einer schnellen und wirksamen Trinkwassersterilisierung nicht als von vornherein aussichtslos aufgegeben werden; denn nur dadurch, daB wir auch dem einzelnen, von seiner Truppe getrennten Manne ein Mittel an die Hand 
geben können, mit dem er sich jederzeit schnell und in einfachster und zuverlässigster Weise ein einwandfreies Trinkwasser bereiten kann, werden wir in der für die Bekämpfung der Heeresseuchen so überaus wichtigen Frage der rationellen Wasserversorgung im Felde neue Erfolge erwarten dürfen".

Auf Anregung von Herrn Geheimrat Prof. Dr. Flügge habe ich daher versucht, eine Methode zu finden, welche auch kleinste Truppenverbände in die Lage versetzt, sich selbst an Ort und Stelle in kürzester Zeit ein schmackhaftes, bekömmliches und jede Infektionsgefahr ausschlieBendes Trinkwasser zu bereiten.

Mit Recht verlangt Hetsch von einem in der Praxis brauchbaren Wassersterilisationsverfahren, daB es bei kurzer Einwirkungsdauer die für den Menschen pathogenen Mikroorganismen, welche durch Trinkwasser verbreitet werden können - in erster Linie also Typhus- und Ruhrbazillen sowie Choleravibrionen - sicher vernichtet, das Wasser in seinem Aussehen, Geschmack und Geruch nicht wesentlich beeinfluBt und in demselben keine Substanzen zurückläßt; welche auch beim Genuß größerer Quantitäten für die Gesundheit irgendwie schädlich sind. Zudem soll das Verfahren einfach und leicht anwendbar sein, die benötigten Ingredienzien sollen lange Zeit in unverändertem Zustande haltbar, nicht teuer sein und beim Transport nicht viel Raum beanspruchen.

Wenn in den zahlreichen Arbeiten, welche sich mit der Frage der chemischen bzw. physikalischen Trinkwassersterilisation beschäftigen, häufig so verschiedene Resultate erzielt worden sind, so liegt die Ursache hauptsächlich darin, daB das Material, an welchem das Verfahren geprüft wurde, verschieden war und daher bald gröBere, bald geringere Anforderungen an das Sterilisationsverfahren stellte. Die Schwierigkeit derartiger Prüfungen beginnt eben bereits bei Beantwortung der Frage, welche Mengen von künstlicher Bakterieneinsaat und welche chemische und physikalische Beschaffenheit des Wassers wir wählen müssen, um den schwierigsten in der Natur vorkommenden VerhäItnissen Rechnung zu tragen. Wenn auch erfahrungsgemäB die Krankheitserreger zum gröBten Teil im Wasser schnell zugrunde gehen, so muß doch in der Praxis damit gerechnet werden, daB z. B. Typhusdejekte kurze Zeit vor der Entnahme in das Wasser gelangt sind. Andererseits kann der Gehalt des zu desinfizierenden Wassers an organischer Substanz für den Desinfektionseffekt von ausschlaggebender Bedeutung sein, indem durch Oxydation derselben ein Teil des Desinfektionsmittels verbraucht wird. Vor allem aber nehmen die im Wasser vorhandenen Schwebeteilchen rein physikalisch einen Teil des Desinfektionsmittels infolge der eintretenden Oberflächenspannung so sehr in Beschlag, daB die physikalische Beschaffen- 
heit des zu sterilisierenden Wassers die wichtigste Rolle spielt. Wiederholt habe ich mich in Versuchen davon überzeugen können, daB man z. B. mit dem Schumburgschen Bromverfahren wesentlich bessere Resultate erzielt, wenn man Spreewasser vor Einbringen der Colikeime durch Zusatz von schwefelsaurer Tonerde mit nachfolgender Filtration vorklärt, indem nun die Balterien als einzig vorhandene Formelemente das Desinfektionsimittel infolge Oberflächenanziehung auf sich konzentrieren.

Um nun meine Versuchsanordnung von vornherein so schwierig $\mathrm{zu}$ gestalten, dab sie auch praktischen Forderungen standhielt, benutzte ich bei allen Versuchen Spreewasser, welches in der Nähe der Marschallbrücke, wo stets Schiffe lagern, jedesmal frisch entnommen wurde und außer massenhaften Schwebeteilchen bereits 20000 bis 100000 Keime im Kubikzentimeter enthielt, und versetzte dasselbe noch pro Liter mit einer 24 stündigen, unfiltrierten Schrägagarkultur (etwa 750000000 Keime) von Bacterium coli, einer Einsaat, welche diejenige, an der Hetsch die chemischen Verfahren nachprüfte, ohne ein praktisch brauchbares zu finden, um das 10 bis 100 fache übertrifft. Das derart verunreinigte Wasser enthielt so viel organische Substanz, daß $20 \mathrm{mg}$ Sauerstoff pro Liter zur Oxydation erforderlich waren. Diese Sauerstoffmenge entspricht einem Gehalt von $400 \mathrm{mg}$ organischer Substanz im Liter, während brauchbares Grundwasser z. B. im Maximum nur $40^{\mathrm{mg}}$ organischer Substanz pro Liter enthält. Mit einem derart verunreinigten Wasser dürfite also wohl auch den in der Natur zu erwartenden, schwierigsten Verhältnissen so weit Rechnung getragen sein, daß ein an solchem Testmaterial geprüftes Verfahren den strengsten Anforderungen Genüge leistet, welche man an ein Desinfektionsverfahren stellen kann.

Welche Anforderungen nun dieses Testmaterial an ein Desinfektionsmittel stellt, prüfte ich zunächst an dem Schumburgschen Bromverfahren und zwar mit den Reagentien der Oranienapotheke nach der von Schüder geforderten Versuchsanordnung. Selbst die vierfache der von Schumburg angegebenen Bromdosis $(0.24 \mathrm{~cm}$ pro Liter) vermochte auch in der doppelten Zeit (10 Minuten) nicht die Colikeime abzutöten. Erst wenn ich das mit Bacterium coli infizierte Spreewasser in der oben angegebenen Weise vorklärte, trat der Desinfektionseffekt zutage. Der Erfolg war jedoch immer noch unsicher. So töteten $0.12 \mathrm{~cm}$ Brom pro Liter in 10 Minuten dreimal die eingebrachten Colikeime $a b$, fünfmal nicht. Erst $0.18 \mathrm{~cm}$ Brom pro Liter vernichteten in 10 Minuten oder $0.24 \mathrm{~cm}$ Brom pro Liter in 5 Minuten in acht angesteliten Versuchen alle Colikeime. Annähernd den gleichen Effekt erzielte ich jedoch, wie erwähnt, auch dann, wenn ich das Spreewasser nicht nach, sondern vor Einbringen der Colikeime vorklärte. In je acht derart angestellten Versuchen trat 
$1 \mathrm{mal}$ bei 0.12 com Brom und 10 Minuten langer Einwirkung

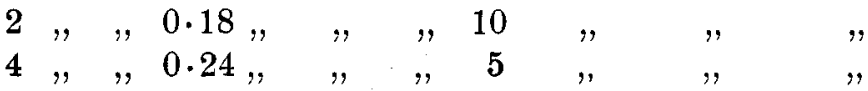

keine Keimentwicklung mehr ein. Da die Versuchsanordnung in beiden Versuchsreihen sonst absolut gleich war, glaube ich dieses Resultat mit Recht darauf zurückführen zu können, daB bereits das Entfernen der Schwebeteilchen für das Desinfektionsmittel günstigere Bedingungen schafft.

Auf Grund dieser Versuche und der zahlreichen in der Literatur angegebenen, ${ }^{1}$ aber nicht anerkannten Verfahren, sei es nun, da $\beta$ es sich um Filtration oder Desinfeltion handelte, kam ich zu der Vorstellung, daß das Ziel einer schnellen Bereitung keimfreien Trinkwassers nur durch eine Vereinigung beider Verfahren zu erreichen sei.

Man kann wohl hoffen, in der kurzen Zeit von 10 bis 15 Minuten (längere Zeit kommt praktisch nicht in Frage) die einzelnen oder in kleinen Verbänden im Wasser suspendierten Miliroorganismen auf chemischem Wege abzutöten, es scheint aber von vornherein aussichtslos, auf diese Weise auch an die Keime heranzukommen, welche geschützt im Innern von Stuhlpartikelchen liegen oder von sonstigen Substanzen eingehüllt sind. Andererseits sind es bei der Filtration vermutlich gerade wieder die freischwebenden Mikroorganismen, welche durch die Filterporen hindurchschlüpfen, während die zu Klümpchen geballten oder in Klümpchen eingehüllten Keime leichter zurückgehalten werden. Von einer Filtration des Wassers wird man aber in den meisten Fällen sowieso nicht absehen können, weil das verdächtige Wasser trübe ist; denn dem Soldaten wird sich von vornherein mehr das Wasser empfehlen, welches klar und appetitlich aussieht, als das, welches zwar keimfrei sein soll, aber trübe ist und unappetitlich wirkt. Bei Anwendung chemischer Mittel ist andererseits so gut wie sicher damit zu rechnen, daB Niederschläge im Wasser auftreten, welche dann doch eine Filtration erfordern. Da eine doppelte Filtration aber schon durch den Mangel an Zeit verboten wird, ist von einem praktisch brauchbaren Verfahren zu verlangen, daB es durch Zusatz eines chemischen Mittels in das rohe, nicht vorgeklärte Wasser 1. die freischwebenden, bei der nachfolgenden Filtration durchschlüpfenden Mikroorganismen abtötet; und 2. die durch die chemische Desinfektion nicht abgetöteten Keimklümpchen sicher einhüllt und so auf dem Filter zurückhält.

1 Eine sehr vollständige Übersicht der Wasserreinigungsverfahren gibt Selberg in der Vierteljahrssểrift für gerichtliche Medizin. 1913. Bd. XLVI. Hft. 2 u. 3. 
Filtrierverfahren: Da der einzige in die Armee eingeführte Filter, der ron Eben konstruierte Berkefeld-Armeefilter, abgesehen von seiner Durchlässigkeit für Keime, wegen seiner Empfindlichkeit, seinem Versagen bei stärker getrübtem Wasser, seiner umständlichen Reinigung und der dauernd notwendigen bakteriologischen Kontrolle für Feldverhältnisse nicht geeignet ist, wie der südwestafrikanische Feldzug zur Genüge bewiesen hat, sảh ich mich zunächst nach einem brauchbaren Filtergewebe um und wurde dabei auf einen Trinkwasserfilter aufmerksam, welcher als Haushaltungsfilter gute Dienste leisten sollte und, nach dem Gewebe des Filteriörpers zu schlieBen, für Feldverhältnisse gut geeignet schien. Es handelt sich um die sogenannnten Sucrofilter, deren Filterkörper aus einem Asbestgewebe besteht, welches nach einem patentierten Verfahren mit einem, im wesentlichen aus Marmor, Asbestwolle und Silikaten hergestellten Brei in der Hitze bei $200^{\circ}$ imprägniert wird. Da es also einerseits möglich schien, die Sterilisation der Filterkörper, nachdem sie durch Ausbürsten gereinigt sind, einfach durch Ausglühen am offenen Feuer zu bewerkstelligen, und andererseits eine Zerstörung des Filtergewebes selbst bei den unvermeidlichen Insuiten im Felde - nicht so leicht zu befürchten war, prüfte ich diese Sucrofilter auf ihr Wasserreinigungsvermögen. Das Ergebnis dieser Untersuchungen war durchaus ermutigend. Spreewasser, welches in der angegebenen Weise mit Bacterium coli infiziert und dadurch stark getrübt wurde, lief, durch den Sucrofilter gegossen, kristallklar ab und zeigte auch eine ganz erhebliche Keimverminderung; durchschnittlich ging von 4000 Keimen erst einer durch das Filtergewebe hindurch.

Da ein bei der Firma vorhandenes Armeefiltermodell für Feldverhältnisse durchaus ungeeignet war, ging ich zunächst daran, einen diesen Verhältnissen angepaßten Filter zu konstruieren. Die von der Firma bisher angefertigten Apparate waren als Reservoirfilter gedacht und trugen am Filterkörper daher neben dem unten befindlichen AusfluBrohr für das filtrierte Wasser noch ein nach oben über die Wasserfläche hinausgeführtes Luftrohr, um eine Ansammlung des filtrierten Wassers bei geöffnetem Lufthahn zu ermöglichen, selbst wenn der Wasserausflußhahn geschlossen war. Durch dieses Luftrohr war aber erstens die Gefahr einer Infektion des Filterinneren vorhanden und auBerdem war die Filterleistung beschränkt, da die Höhe des Spiegels des zu filtrierenden Wassers durch den Hahn am Luftrohr vorgeschrieben wurde. Da es sich unter Feldverhältnissen nur darum handelt, im Bedarfsfalle zu filtrieren, aber nicht ein Wasserreservoir mitzuführen, lieB ich das Luftrohr fort und auch den Hahn am WasserausfluBrohr, welches ich so weit machen lieB, daB die von dem Wasser aus dem Filterinneren zu verdrängende Luft mit dem Wasser 
zugleich aus demselben abströmen konnte. Hierdurch gewann ich nun zugleich die Möglichkeit, durch Verlängerung des Wasseraufnahmegefäßes nach oben über den Filterkörper binaus die Filterleistung zu erhöhen, da ich jetzt mit dem Druck einer Wassersäule von etwa $20^{\mathrm{cm}}$ arbeiten konnte. In diesem Zustande war der Filter jedoch nicht transportabel, so daB es nötig wurde, die Verlängerung durch einen teleskopartig ausziehbaren Blechzylinder zu ersetzen. Da bei ovaler Form des Gefäßes

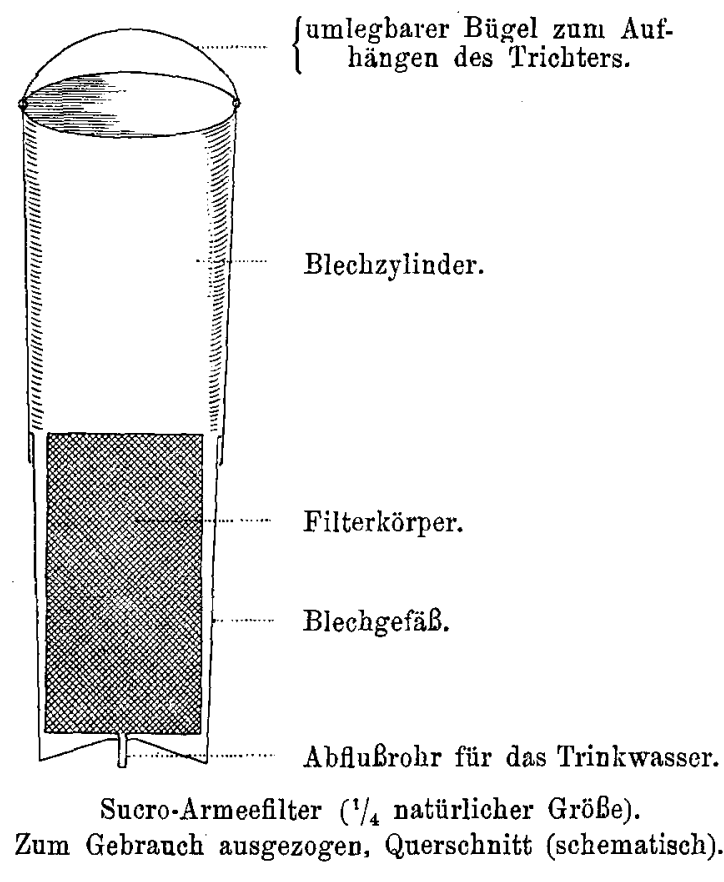

das wasserdichte Anliegen des ausgezogenen Zylinders nicht zu garantieren war, mußte ich eine runde Form für den Filter wählen.

Der aus diesen Versuchen resultierende Armeefilter hat sich unter der Praxis angepabten Verhältnissen bewährt. Er ist so abgemessen, daß ex in einem runden Kochgeschirr von $22 \mathrm{~cm}$ Höhe und $11^{\mathrm{em}}$ Durchmesser untergebracht werden kann. Er besteht aus einem runden Blechgefäb, welches am Boden festgeschraubt, den Filterkörper enthält. Das BlechgefäB wird von einem Blechzylinder umschlossen, welcher demselben so angepaBt ist, daB er beim Herausziehen das BlechgefäB wasserdicht abschließend, dieses um $20^{\mathrm{cm}}$ nach oben verlängert und so einen Behälter zur Aufnahme des zu filtrierenden Wassers schafft. 
Der Filter wiegt, aus Aluminiumblech hergestellt, $1 \mathrm{~kg}$ und liefert bei der Beschickung mit dem infizierten Spreewasser bei ständigem Nachgießen in 5 Minuten 1 bis $1^{1 /} / 2$ Liter kristallklares Wasser. Diese Filterleistung wird sich bei Anfertigung der Filterkörper im großen noch erhöhen, da es dann technisch möglich ist, die Filterkörper aus einem Stück (strumpfartig) zu weben, so daB die bei dem Probemodell noch vorhandenen Nähte fortfallen. Die Filterfläche wird dadurch um etwa $100^{\text {qem }}$ größer.

Diè Keimzahl des Filtrates schwankt wie bei allen Filtern, je nachdem der Filter frisch gereinigt ist oder schon längere Zeit im Gebrauch war, wodurch bei geringerer Ergiebigkeit niedrigere Keimzahlen erzielt werden. Ein keimfreies Filtrat wurde jedoch nie erreicht und zwar aucb dann nicht, wenn ich die Filterkraft durch Zusatz von Fällungsmitteln noch erhöhte. AuBer den bekannten Substanzen, wie Aluminiumsulfat, Calcium- und Eisenverbindungen benutzte ich auch geschlemmte Kieselgurerde und ein von einer österreichischen Ceresinfabrik empfohlenes Mittel, welches eine stark bakterienbindende Kraft besitzen sollte und den Namen „Macanit" führt. Zwar gelingt es auf diese Weise unter mehr oder weniger herabgesetzter Ergiebigkeit die Keimdichtigkeit der Filtermasse so zu erhöhen, daß man auch einmal in $1^{\mathrm{ccm}}$ des Filtrates keinen Keim findet, aber bei einer Probe von $100^{\mathrm{ccm}}$ und Anreicherung habe ich stets Keime nachweisen können.

Die Versuche, einfach durch eine Filtration zu einem keimfreien Trinkwasser au gelangen, führten also, wie auch nicht anders zu erwarten war, zu keinem Resultat.

Chemisches Verfahren: Daher versuchte ich nunmehr ein chemisches Mittel zu finden, welches, wenn auch nicht die in Klümpchen eingehüllten Keime, so doch wenigstens diejenigen mit Sicherheit in meinem infizierten Spreewasser abtöten sollte, welche, weil freis $\mathrm{ch}$ w ebend, bei einer nachfolgenden Filtration nicht zurückgehalten würden.

Da sich bei den von Hetsch angestellten Nachprüfungen der zahlreichen empfohlenen chemischen Präparate nur noch das Wasserstoffsuperoxyd einigermaßen bewährt hatte, entschlob ich mich dazu, dieses gleichfalls zu prüfen, vor allem auch, weil ich auf ein neues Präparat aufmerksam geworden war, welches von der Firma Bayer \& Co (Elberfeld) hergestellt wird. Dasselbe ist ein festes Wasserstoffsuperoxyd ( 36 prozentig) und zwar eine Additionsrerbindung von Carbamid und Wasserstoffsuperoxyd, Ozuron genannt. Ich suchte zunächst festzustellen, wie sich dieses Präparat den von mir angewandten Einsaatmengen gegenüber bei 5 bzw. 10 Minuten dauernder Einwirkung verhielt. Zu diesem Zwecke verteilte ich 1 Liter Spreewasser + eine Kultur Bakterium coli auf 20 Erlenmeyer- 
kölbchen so, daß jedes Kölbchen $50^{\mathrm{cem}}$ enthielt. Dann fügte ich von einer 6 prozentigen, aus dem Ozuron hergestellten Wasserstoffsuperoxydlösung so viel in die einzelnen Kölbchen hinzu, daß ich 1 promillige bis 1 prozentige Lösungen bekam und zwar von jeder Konzentration 2 Kölbchen. Nach 5 bzw. 10 Minuten wurde das Wasserstoffsuperoxyd dadurch zerstört, daB fein gepulverter, steriler Braunstein $\left(1 \mathrm{grm}\right.$ auf $\left.50^{\mathrm{ccm}}\right)$ hineingeschüttet und durch sterile Papierfilter filtriert wurde. Durch Titration mit Kaliumpermanganatlösung ( $1 / 10$ n.) überzeugte ich mich, daB auch aus der stärksten Lösung alles Wasserstoffsuperoxyd vernichtet war. Von dem Filtrat wurde $1^{\mathrm{cem}}$ zur Gelatineplatte verarbeitet, und die Platten nach 8 Tagen ausgezählt. Das Resultat ergibt folgende Tabelle:

\begin{tabular}{|c|c|c|c|c|c|c|c|c|c|c|}
\hline \multirow{2}{*}{$\begin{array}{c}\text { Einwirkungs- } \\
\text { dauer }\end{array}$} & \multicolumn{10}{|c|}{ Wasserstoffsuperoxydlösung } \\
\hline & $1 \%$ & $2 \%$ & $3 \%$ & $4 \%$ & $\begin{array}{l}5 \% \\
\mathrm{Ke}\end{array}$ & $\begin{array}{l}6 \% \\
\mathrm{~m} e\end{array}$ & $7 \%$ & $8{ }_{100}$ & $9 \%$ & $1 \%$ \\
\hline 5 Mint & 10 & 8 & 4 & $\mathbf{1}$ & 1 & 1 & 0 & 0 & 0 & 0 \\
\hline 10 & 18 & 4 & 4 & 2 & 0 & 0 & 0 & 0 & 0 & 0 \\
\hline
\end{tabular}

Danach konnte ich also eine 1 prozentige Wasserstoffsuperox jdlösung bei einer Einwirkungsdauer von 5 Minuten als sicher abtötende Dosis meiner Einsaat gegenüber ansehen, entsprechend der von Traugott stammenden Angabe, daß Typhusbazillen in 1 prozentiger Wasserstoffsuperoxydlösung in 5 Minuten zu Grunde gehen. Eine Konzentration von 1 Prozent hätte aber etwa 30 grm $\mathrm{Ozuron}$ pro Liter gefordert und kam daher praktisch nicht in Frage. Da.jedoch beim Einbringen des Braunsteins auffiel, daß auch in der schwächsten Lösung noch erhebliche Mengen von Wasserstoffsuperoxyd unverbraucht geblieben waren, lag der Gedanke nahe, zu versuchen, ob nicht auch geringere Mengen von Wasserstoffsuperoxyd einen Effekt zeigen würden, wenn es nur gelänge, die Einwirkung desselben auf die Keime zu fördern. Dazu war jedoch zunächst die Frage zu klären, worauf die keimtötende Kraft des Wasserstoffsuperoxyds überhaupt beruht.

Das Wasserstoffsuperoxyd steht chemisch dem Sauerstoff, speziell in seiner aktiven Form als Ozon $\mathrm{O}_{3}$.nahe, da es wie dieses auch ein nur lose gebundenes 0 -Atom besitzt. Während das Ozon jedoch dieses O-Atom schon spontan abgibt, muB es in dem Wasserstoffsuperoxyd erst abgespalten werden, um seine oxydierende bzw. desinfizierende Wirkung ausüben zu können. Diese Zersetzung des Wasserstoffsuperoxyds kann nun jedoch in verschiedener Weise erfolgen. So rufen z. B. einige Superoxyde, wie Braunstein $\left(\mathrm{MnO}_{2}\right)$, eine augenblickliche, stürmische Sauerstoffentwicklung hervor, indem sie das Wasserstoffsuperoxyd nach der Formel

$$
2 \mathrm{H}_{2} \mathrm{O}_{2}=2 \mathrm{H}_{2} \mathrm{O}+\mathrm{O}_{2}
$$


zerlegen. Dieser „Katalyse“ genannte Vorgang wird nun auch durch gewisse Fermente (,Katalase" oder "Superoxydase" nach Raudnitz) ausgelöst, welche nach den Untersuchungen Jorns auch von den Bakterien produziert werden. Diese Fermente haben demnach die Aufgabe, aus den Peroxyden den molekularen Sauerstoff abzuspalten und so der Zelle zur Verfügung zu stellen oder auch einen schädlichen ÜberschuB an Peroxyden auf diese Weise im Notfalle sofort zu beseitigen, um das lebende Zellprotoplasma zu schützen. Von dieser katalytischen Wirliung der Bakterien kann man sich in der Weise leicht überzeugen, daB man eine Aufschwemmung derselben zu einer Wasserstoffsuperoxydlösung hinzugibt. Man bèobachtet dann in der Lösung eine mehr oder weniger starke Gasbildung. Der molekulare d. h. inaktive Sauerstoff 0-0 übt aber auch in statu nascendi keine keimtötende. Wirkung ans. Zerstört man nämlich sofort nach dem Einbringen das Wasserstoffsuperoxyd wieder durch Braunstein, wobei der ganze aus demselben zu erhaltende Sauerstoff frei wird, wodurch die Flüssigkeit stark aufschäumt, so findet keine merkliche Abtötung der Keime statt. Die desinfizierende Wirkung des Wasserstoffsuperoxyds ist demnach nur so $\mathrm{zu}$ denken, daB dasselbe, im UeberschuB vorhanden, zunächst die Bakterienkatalase absättigt, sich dann an der Oberfiäche der Bakterien konzentriert und nun durch Abspaltung von atomistischem Sauerstoff 0 nach der Formel

$$
\mathrm{H}_{2} \mathrm{O}_{2}=\mathrm{H}_{2} \mathrm{O}+\mathrm{O}
$$

eine chemische Wirkung auf das Protoplasma ausübt.

Eine Unterstützung der Desinfektionswirkung des Wasserstoffsuperoxyds wäre also in der Weise denkbar, daß man diese Abspaltung atomistischen Sauerstoffs in unmittelbarer Verbindung mit der Zelle förderte.

$\mathrm{Zu}$ einer derartigen Beschleunigung eines chemischen Vorganges konnten die in der Chemie als Katalysatoren bekannten Stoffe geeignet sein, sofern sie nicht wie Braunstein z. B. die Zerlegung des Wassersuperoxyds in molekularen Sauerstoff bewirken. Das auch normulerweise im Wasser vorkommende schwefelsaure Eisenoxydul $\left(\mathrm{FeSO}_{4}\right)$ schien mir für meine Zwecke am geeignetsten, da es im Wasser leicht lüslich ist und beim Zusatz oxydierender Mittel in unlösliches basisches Ferrisulfat übergeht. Da nach Gottstein die Nukleoalbumine durch Wasserstoffsuperoxyd besonders leicht oxydiert werden, schien es möglich, daß das unter der Einwirkung des schwefelsauren Eisenoxyduls aus dem Wasserstoffsuperoxyd sich abspaltende O-Atom in statu nascendi die Bakterien leichter angreift als die anorganische Substanz.

In 50 nach dieser Richtung hin angestellten Versuchen habe ich eine Bestätigung dieser Vermutung erfahren, wenn auch nicht bis zu dem Grade, daß eine Verwendung des Verfahrens in der Praxis möglich wurde. 
Denn wenn auch der Filterrückstand stets Colikeime enthielt, so konnte ich doch in 12 Prozent der Versuche auch mit Hilfe des Anreicherungsverfahrens im Filtrat keine Colikeime mehr nachweisen. Dieser Erfolg, den ich bei meinen Filtrationsversuchen früher nie erreichte, ist also sicher nicht nur auf physikalische Einflüsse (Niederschlagswirkung) zurückzuführen, sondern auf eine Oxydationswirkung des Wasserstoffsuperoxyds, obgleich ich dasselbe nur in 0.4 bis 0.8 promilliger statt in 1 prozentiger Lösung anwandte, aber 1 bis 2 grm Ferrosulfat pro Liter zusetzte; die Einwirkungsdauer betrug bei allen Versuchen zehn Minuten. Der bereits bei den angewandten Mengen von Ferrosulfat im Filtrat auftretende Eisengeschmack verbot die auch sonst wenig aussichtsreiche Anwendung höherer Dosen, so daB ich die Versuche abbrach.

Es blieb nun noch ein Weg, welcher zum Ziele führen konnte, indem man auf das Wasserstoffsuperoxyd als Desinfektionsmittel verzichtete und nur seine Eigenschaft als kräftiges Oxydations- und Reduktionsmittel benutzte, um andere Desinfektionsmittel nach stattgehabter Einwirkung wieder zu vernichten.

Mit Chlorkalk und Jodtrichlorid angestellte Versuche versagten, da es nicht gelang, das Desinfektionsmittel restlos aus dem Wasser zu entfernen. Auch in den günstigsten Fällen blieb ein leichter Arzneigeschmack zurück, welcher den GenuB des Wassers verhinderte. Der Chlorkalk ist überhaupt wegen seines wechselnden Chlorgehaltes und seiner schweren Löslichkeit ein sehr fragliches Wasserdesinfektionsmittel und versagte den von mir angewandten Einsaatmengen gegenüber in den praktisch möglichen Konzentrationen durchaus, während das Jodtrichlorid in 2 promilliger Lösung gut desinfizierte, aber dem Wasser einen nicht zu beseitigenden Arzneigeschmack verlieh.

Da ich bei meinen Titrationen der Wasserstoffsuperoxydlösungen eine Kaliumpermanganatlösung benutzte, lag der Gedanke nahe, auch dieses Präparat zur Wasserdesinfeltion heranzuziehen, um es nachträglich durch Wasserstoffsuperoxyd zu zerstören.

Das Kaliumpermanganat wird ja bereits mit gutem Erfolg zur Vorklärung benutzt; eine vollständige Sterilisierung von Oberflächenwasser war aber nur mit großen Mengen und mehrtägiger Behandlung zu erzielen, so daB dem Verfahren eine praktische Bedeutung bisher nicht zukam, auch nicht in der von französischer Seite empfohlenen Kombination mit Eisen- oder Mangansulfat, welcher auch Glaser speziell für militärische Zwecke das Wort redet. Die Desinfektionswirkung des Kaliumpermanganats beruht auf seiner Abgabe von Sauerstoff und der dabei stattfindenden Umsetzung zu Braunstein, welcher mechanisch fällend wirkt, so daß bis 98 Prozent der Keime aus dem Wasser verschwinden sollen (Reichel). 
Nach anfänglich in bakteriologischer Beziehung nicht befriedigenden Resultaten gelang es mir durch Kombination des Kaliumpermanganats mit dem wiederum als Katalysator wirkenden Ferrosulfat zu gleichen Teilen bei einem Zusatz von $1{ }^{\mathrm{grm}}$ pro Liter und 10 Minuten dauernder Einwirkung ein klares, schmackhaftes und colifreies Wasser zu erzielen, nach dem ich die angewandten Chemikalien durch 1 grm festes Wasserstoffsuperoxyd pro Liter $(0.36$ Prozent) in Braunstein und unlösliches Ferrisulfat übergeführt und filtriert hatte. Da auch der auf dem Filter zurückbleibende Schlamm keine Colientwicklung zeigte, wenn ich ihn direkt auf Endoplatten verarbeitete, glaubte ich bereits mein Ziel erreicht zu haben, zumal auch der mit Bouillon geschüttelte Schlamm beim Ausstreichen der stark getrübten Bouillon auf Endoplatten keine Colikeime zur Entwicklung kommen ließ. Wenn ich aber den mit der Bouillon geschüttelten Schlamm erst 24 Stunden im Brutschrank bei $37^{\circ}$ gehalten hatte, konnte ich stets noch Colikeime auf der Endoplatte nachweisen. Es handelte sich also nur um eine, wenn auch sehr sichere Einhüllung der im Schlamm zurückgebliebenen Keime in den Niederschlag von Braunstein und Ferrisulfat, ohne daß jedoch der Schlamm in der Lage gewesen wäre, die eingehüllten Keime abzutöten. Wie sicher diese Einhüllung jedoch war, davon konnte ich mich dadurch überzeugen, daB ich das unfiltrierte Wasser 24 Stunden lang mit dem Schlamm in Berührung lieb, ohne dab Keime aus demselben in das Wasser übergingen.

$\mathrm{DaB}$ die in dem Schlamm in entwicklungsfähigem Zustande zurückbleibenden Keime nicht unbedenklich waren, stellte sich sofort heraus, als ich den Sucro-Armeefilter zur Filtration benutzte und, ohne ihn zu reinigen, 24 Stunden lang stehen lieB, um ihn dann in diesem Zustande zu einer neuen Filtration zu benutzen. Das Filtrat war dann nicht mehr colifrei, d. h. es hatte eine Infektion des Filters stattgefunden. Es durfte also unter keinen Umständen auf die Forderung verzichtet werden, auch den auf dem Filter zurückbleibenden Schlamm colifrei zu machen oder, wenn dies, was anzunehmen war, in der kurzen Zeit von 10 Minuten nicht gelang, wenigstens ein keimhemmendes Mittel in den Schlamm hinein zu bekommen, welches die Keime allmählich absterben lieb und so eine Infektion des Filtergewebes verhinderte.

Dies letztere gelang mir endlich, als ich das Ferrosulfat durch das gleichfalls katalytisch wirkende Kupfersulfat ersetzte, da nun die im Schlamm zurückbleibenden Kupferverbindungen die darin eingeschlossenen Keime allmählich abtöteten.

Um eine Nachprüfung dieser Versuche zu ermöglichen, werde ich meine Versuchsanordnung sowie die Ergebnisse eingehend besprechen und letztere der Utbersicht halber in einer Tabelle zusammenstellen. 


\section{Versuchsanordnung.}

4 Liter frisch entnommenes Spreewasser werden mit 4 Schrägagarröhrchen einer 24 stündigen Kultur von Bacterium coli versetzt, nachdem diese mit Spreewasser abgeschwemmt und in einem Erlenmeyerkölbchen kräftig durchgeschüttelt sind. Das so infizierte Spreewasser bleibt eine Stunde bei Zimmertemperatur stehen, um eine den natürlichen Verhältnissen möglichst angepaßte Verteilung der Colikeime im Wasser zu erreichen. Dann werden die Desinfektionsmittel in Pulverform dem Wasser zugesetzt und zwar zunächst $3^{\mathrm{grm}}$ Kupfersulfat und nach kräftigem Umschütteln $3^{\mathrm{grm}}$ Kalium. permanganat. Dieses löst sich wesentlich langsamer als Kupfersulfat, so daB es erforderlich ist, das Wasser während der Desinfektionszeit vor allem.in den ersten Minuten in Bewegung zu halten. Nach Ablauf von 10 Minuten werden vier Tabletten à 1 grm festen 36 prozentigen Wasserstoffsuperoxyds hinzugetan und wiederum während der etwa 3 Minuten dauernden Zerstörung der Desinfizientien mehrmals kräftig geschüttelt. Die anfangs rotviolette Farbe der Flüssigkeit geht dabei allmählich in ein deutliches Braun über und beweist so, daB alles Kupfersulfat und Kaliumpermanganat zerstört ist. Der Soldat hat also in diesem auch für das ungeübte Auge leicht wahrnehmbaren Farbenumschlag ein untrügliches Zeichen, daß alle gesundheitsschädlichen Substanzen aus dem Wasser entfernt sind. Zugleich mit dem Farbenumschlag tritt in dem Wasser ein dichter voluminöser Niederschlag auf, welcher sich sehr schnell zu Boden senkt. Nunmehr wird das Wasser durch den vorher im Autoklaven sterilisierten Sucro-Filter gegossen und läuft, nachdem das erste Wasser die im Filtergewebe infolge der voraufgegangenen Erhitzung veraschten Rückstände herausgeschwemmt hat, kristallklar im Strahl ab. Die Filterleistung, welche sich bei ständigem Nachgießen nicht wesentlich ändert, beträgt in 4 bis 5 Minuten einen Liter. Das abgelaufene Wasser, auch der erste Strahl, wird halbliterweise aufgefangen und durch Zusatz stark alkalischer 10 prozentiger Traubenzuckerpeptonlösung in sehwach alkalische 1 prozentige Traubenzuckerpeptonlösung verwandelt und 24 Stunden bei $37^{\circ}$ bebrütet. Von jedem der bebrüteten Peptonwasserkolben wird dann eine große Platinöse. voll auf Endoplatten ausgestrichen, und das Resultat nach 24 stündigem Aufenthalt der Platten im Brutschrank verwertet. Außerdem werden viermal je drei große Platinösen des auf dem Filterkörper zurückgebliebenen Schlammes in Bouillonröhrchen gebracht, kräftig geschüttelt und nach 24 stündigem Bebrüten gleichfalls mittels Endoplatten auf Bacterium coli geprüft. Um absolut sicher zu sein, daB in dem Filtrat keine Stoffe mehr vorhanden waren, welche keimhemmend wirken konnten, prüfte ich das Filtrat jedesmal auf seinen Gehalt an Wasserstoffsuperoxyd, da sich bei meinen Versuchen herausgestellt hatte, daß die spontane Zersetzung des Wasserstoffsuperoxyds in den Nähr. böden keineswegs so schnell erfolgt, wie einige Autoren (Schmidt, Croner) annehmen. Ich konnte mehrfach durch vergleichende Titrationen mit Kaliumpermanganatlösungen nachweisen, dab in einer Lösung, welche auBer der Bakterieneinsaat 0.5 promilligen Wasserstoffsuperoxyd und 1 prozentigen Traubenzuckerpepton enthielt, noch nach 24 Stunden $3 / 4$ des Wasserstoffsuperoxyds vorhanden waren. Derselbe kann also auch in der an organischer Substanz reichen Nährlösung sich längere Zeit unzersetzt erhalten und zwar 
in einer Konzentration, welche noch energisch hemmend auf das Bacteriumwachstum einwirkt. Denn eine 0.1 promillige Wasserstoffsuperoxydlösung verhindert noch jede Keimentwicklung, erst in 0.5 promilligen Lösungen gelingt es Colikeime zum Wachstum zu bringen. Bei meiner Versuchsanordnung trifft es sich daher außerordentlich günstig, daB das als Neutralisationsmittel für die Desinfizientien dienende Wasserstoffsuperoxyd durch den entstehenden Braunstein zerstört wird, so daB es unbedenklich im $\ddot{\mathrm{U}} \mathrm{b}$ erschuB zugesetzt werden kann, ein Vorteil, welchen kein anderes chemisches Verfahren besitzt. Wie groß dieser Vorteil ist, geht aus folgender Erwägung hervor, deren Richtigkeit sich in zahlreichen Versuchen ergeben hat. Bei dem wechselnden Gehalt des zu desinfizierenden Wassers an organischer Substanz im allgemeinen und Bakterien im besonderen, ist es durchaus notwendig, mit einem Sicherheitszuschlag zu arbeiten, d. h. also das Desinfektionsmittel im Überschuß zuzusetzen. Da nun ferner der Verbrauch des Desinfektionsmittels von dem Gehalt des Wassers an organischer Substanz abhängig ist, muß auch das Neutralisationsmittel wieder im Überschuß zugegeben werden, um mit Sicherheit alle gesundheitsschädlichen Stoffe zu zerstören, d. h. es muß im Wasser in einer gewissen Konzentration zurüekbleiben und macht damit das Verfahren eben unbrauchbar. Aus diesem Grunde müssen alle die Verfahren schon als praktisch unbrauchbar bezeichnet werden, welche mit einem nach dem $\ddot{A} q u i v a l e n t g e w i c h t$ errechneten Zusatz von Desinfiziens und Neutralisationsmittel arbeiten, da eine derartig genau auf einander eingestellte gegenseitige Absättigung eben nur in der Theorie besteht, wie jeder zu seiner Enttäuschung erfährt, wenn einmal das Desinfektionsmittel, das andere Mal wieder das Neutralisationsmittel im Wasser zurückbleibt.

Bei meiner Versuchsanordnung ist das Neutralisationsmittel an sich unschädlich, verleiht jedoch in höheren Konzentrationen dem Wasser einen laugenhaften Geschmack. Es ist aber, wenn überhaupt, nur in Spuren im Filtrat nachzuweisen, welche mit 0.05 Promille weit unter der Schmeckbarkeitsgrenze liegen und, wie erwähnt, auch keine bakterienschädigenden Einflüsse mehr ausüben können. Zur Sicherheit impfte ich jedoch bei jedem Versuch ein Erlenmeyerkölbchen von dem zur Peptonlösung umgewandelten Filtrat mit einer Nadelspitze voll Bacterium coli, welches sich stets reichlich vermehrte.

Versuchsergebnisse.

Die in nachfolgender Tabelle zusammengestellten 38 Versuche veranschaulichen den Gang der Untersuchung und ermöglichen die erzielten Ergebnisse auch auf ihren praktischen Wert hin zu prüfen. Zur Erklärung der angewandten Zeichen und Abkürzungen bemerke ich folgendes: 
W. bedentet, daB die Filtration des Wassers nach dem chemischen Verfahren nur durch einen sterilen Wattebausch erfolgte, welcher in einen Glastrichter hineingepreBt worden war.

$S$. bedeutet den Sucro-Armeefilter; mit einem kleinen $s$ dahinter besagt, dab der Filter zu diesem Versuch frisch im Autoklaven sterilisiert worden war.

$S$. mit einer Zahl dahinter gibt an, zum wievielten Male der Filter benutzt wurde, ohne inzwischen gereinigt worden zu sein.

$+=$ Bacterium coli gewachsen.

$(+)=\underset{\text { schwächt }}{ }, \quad, \quad$, aber in seinen LebensäuBerungen ge-

- = Bacterium coli nicht gewachsen, aber Sporenbildner.

$0=$ steril.

Die ersten 12 Versuche dienten dazu, die zur Abtötung der Colikeime notwendigen Konzentrationen zu ermitteln. Den Wattebausch benutzte ich nur der Einfachheit halber zur Filtration, da ich ja sicher sein konnte, daB der Sucrofilter dieser provisorischen Methode überlegen war. Da es natürlich nicht möglich war, den Wattebausch stets gleich fest zusammenzudrücken, geschah es nicht selten, dab der erste Ablauf nicht völlig klar war. Dann wurde derselbe noch einmal zurũckgegossen, aber in dasselbe GefäB weiter filtriert, so daß etwa im ersten Ablauf mit durchgegangene Keime das Gefäß infizieren mußten.

Das benutzte feste Wasserstoffsuperoxyd wurde mir von drei verschiedenen Firmen zur Verfügung gestellt, bestand jedoch stets, wie erwähnt, aus einer Additionsverbindung von Karbamid und Wasserstoffsuperoxyd mit 36 prozentigem Gehalt an letzterem. Die verwendeten Präparate waren 1. Perhydrit in Tablettenform von der Firma E. Merck, Darmstadt; 2. Hyperol in Tabletten- und Pulverform von der Firma Gedeon Richter, Budapest; 3. das inzwischen in Ortizon umgetaufte Ozuron der Firma Friedr. Bayer \& Co., Elberfeld, in Tabletten- und granulierter Form. Über die Haltbarkeit dieser Präparate äuBern sich die Firmen folgendermaßen:

E. Merck: „Nach den seither gemachten Feststellungen ist das Perhydrit in Substanz und Tablettenform verhältnismāBig sehr lange Zeit haltbar."

Gedeon Richter: „Die Haltbarkeit der Tabletten ist eine unbegrenzte, außer in den Tropengegenden, wo die große Hitze eine geringe Zersetzung herbeiführt." 
Tabelle I.

\begin{tabular}{|c|c|c|c|c|c|c|c|c|c|c|c|c|c|}
\hline 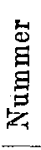 & $\begin{array}{c}\text { Art } \\
\text { des } \\
\text { Wassers }\end{array}$ & 离 & 点蒡 & 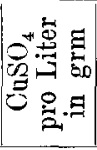 & 吉 & 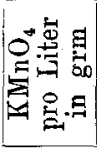 & 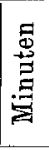 & 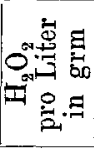 & 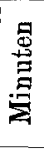 & 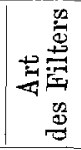 & $\begin{array}{c}\text { An- } \\
\text { gereicherte } \\
\text { Menge }\end{array}$ & 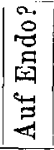 & $\underset{\text { Schlamm? }}{\operatorname{Im}}$ \\
\hline 1 & Spreew. & 1 & $1 \mathrm{C}$ & 0.5 & + & 0.5 & 5 & 0.36 & 2 & $W$. & $1 / 2$ Itr. & - & ++++ \\
\hline 2 & $"$ & 1 & $1 "$ & 0.5 & + & 0.5 & 10 & $0 \cdot 36$ & 2 & $W$. & $"$, & - & +-+0 \\
\hline 3 & $"$ & 1 & 1 & $1 \cdot 0$ & + & $1 \cdot 0$ & 5 & 0.72 & 3 & $W$ & $" \quad "$ & 0 & $-00-$ \\
\hline 4 & $"$ & 1 & $1 "$ & $1 \cdot 0$ & + & $1 \cdot 0$ & 10 & 0.72 & 3 & $W$. & $" \quad$, & 0 & $\begin{array}{lllll}0 & 0 & 0 & 0\end{array}$ \\
\hline 5 & $"$ & 1 & $1 n$ & 1.0 & + & 1.0 & 5 & 0.72 & 3 & $W$ & $" \quad "$ & - & -000 \\
\hline 6 & $"$ & 1 & $1 "$ & 1.0 & + & 1.0 & 10 & 0.72 & 3 & $W$. & $"$, & 0 & $000-$ \\
\hline 7 & $"$ & 2 & 1, & 1.0 & + & $1 \cdot 0$ & 5 & $0 \cdot 72$ & 3 & $W$ & 1, & - & $-\cdots-\cdots$ \\
\hline 8 & $n$ & 2 & $1 "$ & 1.0 & + & $1 \cdot 0$ & 10 & 0.72 & 3 & $W$ & $1 "$ & 0 & $-00-$ \\
\hline 9 & $"$ & 2 & 1, & 0.75 & + & 0.25 & 10 & $0 \cdot 18$ & 2 & $W$ & $1 "$ & + & +-+- \\
\hline 10 & $"$ & 2 & $1 \eta$ & 0.75 & + & 0.5 & 10 & 0.36 & 2 & $W$ & 1 & - & $-\cdots-\cdots$ \\
\hline 11 & $"$ & 2 & $1 "$ & 0.75 & + & 0.75 & 10 & 0.36 & 2 & $W$ & $1 "$ & 0 & $\begin{array}{llll}0 & 0 & 0 & 0\end{array}$ \\
\hline 12 & $"$ & 2 & $1 "$ & 0.75 & + & 0.75 & 10 & 0.36 & 2 & $W$ & 1 & 0 & 0000 \\
\hline 13 & $"$ & 4 & $1 "$ & $0 \cdot 75$ & + & 0.75 & 10 & 0.36 & 3 & S. $s$. & $\begin{array}{l}100 \mathrm{ccm} \\
100 " \\
100 "\end{array}$ & $\begin{array}{l}\overline{0} \\
0\end{array}$ & 00000 \\
\hline 14 & $"$ & 4 & $1 n$ & $0 \cdot 75$ & + & 0.75 & 10 & 0.36 & 3 & S. $s$. & $\begin{array}{cc}1 / 2 & \text { Ltr. } \\
" & n \\
" & " \\
" & "\end{array}$ & $\begin{array}{l}- \\
\overrightarrow{0} \\
0\end{array}$ & $\begin{array}{llll}0 & 0 & 0 & 0\end{array}$ \\
\hline 15 & $"$ & 4 & 1 & 0.75 & + & 0.75 & 10 & 0.36 & $\mathbf{3}$ & S. 1 . & $\begin{array}{ll}n & " \\
" & "\end{array}$ & $\left(\begin{array}{c}- \\
0 \\
(+) \\
0\end{array}\right.$ & $\begin{array}{llll}0 & 0 & 0 & 0\end{array}$ \\
\hline 16 & $"$ & 4 & 1 & $0 \cdot 75$ & + & 0.75 & 10 & $0 \cdot 36$ & 3 & S. 2 . & $\begin{array}{ll}" & " \\
" & " \\
" & " \\
" & "\end{array}$ & $\frac{\overline{0}}{\frac{0}{0}}$ & $\begin{array}{llll}0 & 0 & 0 & 0\end{array}$ \\
\hline 17 & $"$ & 4 & 1, & 0.75 & + & 0.75 & 10 & 0.36 & 3 & S. 3. & $\begin{array}{ll}" & " \\
" & " \\
" & " \\
" & " \\
" & "\end{array}$ & $\left(\begin{array}{c}(+) \\
\frac{0}{0} \\
\frac{0}{0}\end{array}\right.$ & $\begin{array}{llll}0 & 0 & 0 & 0\end{array}$ \\
\hline 18 & $"$ & 4 & 1, & 0.75 & + & 0.75 & 10 & 0.36 & 3 & S. 4. & $\begin{array}{ll}" & " \\
" & " \\
" & " \\
" & " \\
" & "\end{array}$ & $\begin{array}{l}- \\
0 \\
0 \\
0 \\
0\end{array}$ & $\begin{array}{llll}0 & 0 & 0 & 0\end{array}$ \\
\hline 19 & Kanalw. & 4 & 0 & 0.75 & + & 0.75 & 10 & 0.36 & 3 & S. s. & $\begin{array}{ll}n & " \\
" & " \\
" & " \\
" & "\end{array}$ & $\begin{array}{c}- \\
\overline{0} \\
0\end{array}$ & 00000 \\
\hline 20 & $"$ & 4 & 0, & 0.75 & + & $0 \cdot 75$ & 10 & $0 \cdot 36$ & 3 & S. 1. & $\begin{array}{ll}" & " \\
" & "\end{array}$ & $\frac{\overline{0}}{\frac{0}{0}}$ & $\begin{array}{llll}0 & 0 & 0 & 0\end{array}$ \\
\hline
\end{tabular}


Tabelle I. (Fortsetzung.)

\begin{tabular}{|c|c|c|c|c|c|c|c|c|c|c|c|c|c|}
\hline 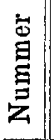 & $\begin{array}{c}\text { Art } \\
\text { des } \\
\text { Wassers }\end{array}$ & 密 & 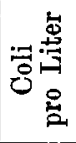 & 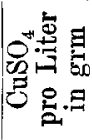 & 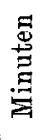 & 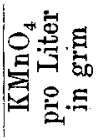 & 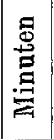 & 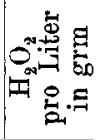 & 焉 & 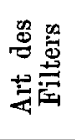 & $\begin{array}{c}\text { An- } \\
\text { gereicherte } \\
\text { Menge }\end{array}$ & 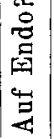 & $\underset{\text { Schlamm? }}{\operatorname{Im}}$ \\
\hline 21 & Kanalw. & 4 & $0 \mathrm{C}$. & 0.75 & + & 0.75 & 10 & 0.36 & 3 & S.2. & $\begin{array}{cc}1 / 2 & \text { Ltr. } \\
" & " \\
" & " \\
" & "\end{array}$ & $\frac{0}{-}$ & 00000 \\
\hline 22 & Spreew. & 4 & 1, & $0 \cdot 75$ & + & 0.75 & 10 & 0.36 & 3 & S. $s$. & $\begin{array}{ll}" & " \\
" & " \\
" & "\end{array}$ & $\frac{-}{0}$ & $\begin{array}{llll}0 & 0 & 0 & 0\end{array}$ \\
\hline 23 & $"$ & 4 & 1, & $0 \cdot 75$ & + & $0 \cdot 75$ & 10 & 0.36 & 3 & S. 1. & $\begin{array}{ll}" & " \\
" & " \\
" & "\end{array}$ & $\begin{array}{c}0 \\
(+) \\
0 \\
0\end{array}$ & $\begin{array}{llll}0 & 0 & 0 & 0\end{array}$ \\
\hline 24 & $"$ & 4 & $1 "$ & 0.75 & + & 0.75 & 10 & $0 \cdot 36$ & 3 & S. 2: & $\begin{array}{l}" \quad " \\
" 1 \\
" \quad "\end{array}$ & $\begin{array}{c}- \\
0 \\
0\end{array}$ & 0000 \\
\hline 25 & " & 4 & 1, & 0.75 & + & 0.75 & 10 & $0 \cdot 36$ & 3 & S. 3. & 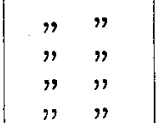 & $\frac{\overline{0}}{0}$ & 00000 \\
\hline 26 & $"$ & 4 & $1 "$ & $0 \cdot 75$ & + & 0.75 & 10 & 0.36 & 3 & S. s. & $\begin{array}{l}" n \\
"\end{array}$ & $\begin{array}{c}- \\
0 \\
0\end{array}$ & 00000 \\
\hline 27 & $"$ & 4 & $1 n$ & 0.75 & + & 0.75 & 10 & $0 \cdot 36$ & 3 & S. 1 . & $\begin{array}{l}", \\
"\end{array}$ & $\begin{array}{l}- \\
0 \\
0 \\
0\end{array}$ & $\begin{array}{llll}0 & 0 & 0 & 0\end{array}$ \\
\hline 28 & $"$ & 4 & $1 "$ & $0 \cdot 75$ & + & $0 \cdot 75$ & 10 & $0 \cdot 36$ & 3 & S. 2. & " " & $\begin{array}{c}- \\
0 \\
0 \\
0\end{array}$ & $\begin{array}{llll}0 & 0 & 0 & 0\end{array}$ \\
\hline 29 & " & 4 & $1 \%$ & 0.75 & + & 0.75 & 10 & $0 \cdot 36$ & 3 & S. 3. & $\begin{array}{l}" n \\
" n \\
" n\end{array}$ & $\frac{-}{0}$ & $\begin{array}{llll}0 & 0 & 0 & 0\end{array}$ \\
\hline 30 & $"$ & 12 & $1 "$ & 0.75 & + & $0 \cdot 75$ & 10 & $0 \cdot 36$ & 3 & S. 4 . & 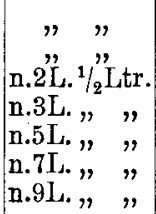 & $\begin{array}{c}- \\
0 \\
0 \\
0 \\
0 \\
0\end{array}$ & 00000 \\
\hline 31 & $"$ & 4 & $1 "$ & $0 \cdot 75$ & 10 & 0.75 & 3 & $0 \cdot 36$ & 3 & S. $s$. & $\begin{array}{cc}1 / 2 & \text { Ltr. } \\
" & " \\
" & " \\
" & "\end{array}$ & $\begin{array}{c}+ \\
+ \\
0 \\
0\end{array}$ & 00000 \\
\hline 32 & $"$ & 4 & $1 "$ & $1 \cdot 0$ & + & $1 \cdot 0$ & 10 & 0.54 & 3 & S. $s$. & $\begin{array}{l}", \\
", \\
"\end{array}$ & $\frac{0}{-}$ & 0000 \\
\hline
\end{tabular}


Tabelle I. (Fortsetzung.)

\begin{tabular}{|c|c|c|c|c|c|c|c|c|c|c|c|c|c|}
\hline 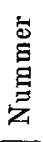 & $\begin{array}{c}\text { Art } \\
\text { des } \\
\text { Wassers }\end{array}$ & $\frac{\dot{9}}{\frac{9}{3}}$ & 语离 & 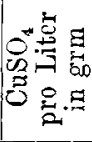 & 苞 & 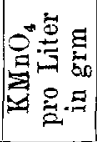 & 胥 & 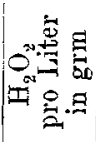 & 晜 & 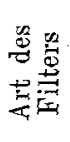 & $\begin{array}{c}\text { An- } \\
\text { gereichert } \\
\text { Menge }\end{array}$ & 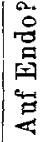 & $\underset{\text { Schlamm? }}{\operatorname{Im}}$ \\
\hline 33 & Spreew. & 4 & $1 \mathrm{C}$. & $1 \cdot 0$ & + & $1 \cdot 0$ & 10 & $0 \cdot 54$ & 3 & S. 1. & $\begin{array}{ll}1 / 2 & \text { Ltr. } \\
" & " \\
" & " \\
" & " 1\end{array}$ & $\begin{array}{l}- \\
0 \\
0 \\
0\end{array}$ & .00000 \\
\hline 34 & $n$ & 4 & $1 n$ & 1.0 & + & $1 \cdot 0$ & 10 & 0.54 & 3 & S. 2. & $\begin{array}{ll}" & " \\
" & " \\
" & " \\
" & ,\end{array}$ & $\frac{0}{-}$ & 0000 \\
\hline 35 & $"$ & 4 & $3 / 4$, & 0.75 & + & 0.75 & 10 & 0.36 & 3 & S. $s$. & $\begin{array}{ll}" & " \\
. & " \\
" & , \\
. & "\end{array}$ & $\begin{array}{l}0 \\
0 \\
0 \\
0\end{array}$ & $\begin{array}{llll}0 & 0 & 0 & 0\end{array}$ \\
\hline 36 & $\because$ & 4 & $3 / 4 "$ & 0.75 & + & 0.75 & 10 & 0.36 & 3 & S. 1. & $\begin{array}{ll}" & , " \\
" & , \\
" & , \\
" & ,\end{array}$ & $\begin{array}{l}- \\
\overline{0} \\
0\end{array}$ & $\begin{array}{llll}0 & 0 & 0 & 0\end{array}$ \\
\hline 37 & $"$ & 4 & $3 / 4 "$ & 0.75 & + & 0.75 & 10 & 0.36 & 3 & S. 2. & $\begin{array}{ll}" & " \\
" & " \\
" & : \\
n & " 1\end{array}$ & $\mid \begin{array}{l}\overline{0} \\
0 \\
0\end{array}$ & 0000 \\
\hline 38 & $"$ & 4 & $3 / 4 "$ & 0.75 & + & 0.75 & 10 & $0 \cdot 36$ & 3 & S. 3. & $\begin{array}{ll}" & " \\
" & " \\
", & " \\
" & "\end{array}$ & $\frac{-}{-}$ & $\begin{array}{llll}0 & 0 & 0 & 0\end{array}$ \\
\hline
\end{tabular}

Friedr. Bayer \& Co. geben ihrem Präparat in granulierter Form den Vorzug und erklären, daB die aus dem Ortizon hergestellten Tabletten zum Schutz vor Feuchtigkeit in Gläsern mit Korkstopfen aufbewahrt werden müssen.

Nach meinen Feststellungen, welche sich über $2^{1 / 2}$ Jahre erstrecken, leisten alle Präparate, gleichviel in welcher Form, in chemischer Beziehung das gleiche. In bezug auf Haltbarkeit standen die Hyperoltabletten an erster Stelle, da sie, nur in Gläsern mit einem Wattebausch verschlossen aufbewahrt, keine Zeichen ron Zersetzung aufwiesen; dasselbe gilt von den Perhydrittabletten, welche jedoch mit Korkstopfen verschlossen waren. Die nur mit einem Wattebausch abgeschlossenen Ortizontabletten waren feucht geworden, hatten jedoch ihre Form behalten.

Kupfersulfat und Kaliumpermanganat sind unbegrenzt haltbar und dürften auch der Verarbeitung zu Tabletten keine Sehwierigkeiten bereiten. Der Ersatz des Kaliumpermanganats durch das viermal so leicht lösliche Calciumpermanganat wird durch die Eigenschaft des letzteren, an der Luft zu zerfließen, leider verhindert. Es käme höchstens in kri- 
stallinischer Form in mit Kork gut verschlossenen Gläsern in Betracht. Zu erwägen wäre ferner noch aus Gründen der Einfachheit das Zusammenreiben von Kupfersulfat und Kaliumpermanganat zu einer Tablette.

Aus den in der Tabelle zusammengestellten Versuchen (1 bis 12) geht hervor, daß bei einer Konzentration von 0.75 Kupfersulfat +0.75 Kaliumpermanganat pro Liter in 10 Minuten langer Einwirkung einmal diejenigen Colikeime, welche die Poren des Wattebausches passieren, mit Sicherheit abgetötet werden, und zweitens die im Schlamm zurückbleibenden Keime nicht mehr zur Entwicklung kommen. Versuch 9 zeigt im besonderen, $\mathrm{da} B$ der Anteil des Kaliumpermanganats an dem Desinfektionseffekt mindestens der gleiche ist wie der des Kupfersulfats. Die schon aus chemischen Gründen, nämlich zur Zerstörung des Kupfersulfats, erforderliche Verwendung der Chemikalien zu gleichen Teilen gibt also auch in bakteriologischer Beziehung das beste Resultat. Dasselbe zeigt der Versuch 31, in welchem zwar die Chemikalien zu gleichen Teilen zugefügt sind, aber verschieden lange einwirken.

Die anderen mit dem Sucro-Armeefilter angestellten Versuche sind durchaus den Feldverhältnissen angepaßt. Die zur ersten Benutzung frisch sterilisierten Filter wurden nämlich, nachdem der Rest des unfiltrierten Wassers fortgegossen war, bis zum nächsten Versuch mit dem Schlamm 24 bis 48 Stunden stehen gelassen und dann von neuem und zwar bis zu vier Malen hintereinander benutzt, ohne inzwischen irgend wie gereinigt oder gar sterilisiert worden zu sein.

Bei dieser Versuchsanordnung hätte also unter allen Umständen eine Infektion des Filtergewebes eintreten müssen, wenn die im Schlamm zurückgebliebenen Keime nicht allmählich abgestorben wären. $\mathrm{DaB}$ bei der Dosis von $0.75^{\mathrm{grm}}$ Kupfersulfat + Kaliumpermanganat $\overline{\mathbf{a a}}$ unter 66 Proben dreimal (Versuch 15, 17 und 23) Colikeime im Filtrat nachweisbar waren, gibt noch keinen Grund die Dosis zu erhöhen, da einmal die Colikeime stark geschwächt waren, wie ihre geringe Säurebildung auf den Endoplatten, ihre schwere Färbbarkeit und ihre verringerte Bewegungsfähigkeit bewiesen, und andererseits selbst im Kanalwasser in drei Versuchen (Nr. 19, 20 und 21) alle Colikeime abgetötet wurden; ein Beweis, welch überaus hohe Anforderungen das von mir angewandte Testmaterial an ein Desinfektionsverfahren stellt. Wie zu erwarten, genügt die Erhöhung der Dosis auf 1.0 grm (Versuch 32, 33, 34) oder die Herabsetzung der Einsaatmenge auf $3 / 4$ Kultur pro Liter (Versuch 35, 36, 37, 38), um das Auftreten selbst dieser geschwächten Colikeime im Filtrat mit Sicherheit zu verhindern.

Auf Grund dieser Versuche darf ich behaupten, daB es bei Anwendung dieses Verfahrens auch unter Feldverhältnissen gelingen muB 
jedes 0 berflächenwasser, das überhaupt nur als Trinkwasser in Frage kommen kann, mit Sicherheit von Colikeimen und damit auch von den in Frage kommenden pathogenen Mikroorganismen zu befreien.

Es erübrigt sich jedoch noch der Nachweis, daß das nach diesem Verfahren gewonnene Wasser auch als Trinkwasser zu benutzen, d. h. geschmacklos ist und keine gesundheitsschädlichen Stoffe enthält. Was den Geschmack betrifft, so kann ich nur die Urteile der Personen wiedergeben, welche das Wasser hier im Institut gekostet haben, wobei ich verschiedene Wasserproben nebeneinander trinken lieB, ohne daB die Versuchspersonen wuBten, welches das nach diesem Verfahren hergestellte Wasser war. Außerdem habe ich selbst das Wasser in lauwarmem Zustande gekostet und kann nur wie alle anderen bezeugen, da $B$ es absolut frei von jedem fremdartigen Beigeschmack ist. Es schmeckt, kühl getrunken, wie Quellwasser und wurde von verschiedenen Personen sogar dem Leitungswasser vorgezogen.

DaB diese Urteile nicht eines sachlichen Grundes entbehren, davon konnte ich mich durch die wiederholt vorgenommene chemische Untersuchung des Wassers überzeugen, welche zugleich auch den Nachweis erbrachte, dab keine gesundheitsschädlichen Substanzen in dem Filtrat vorhanden sind.

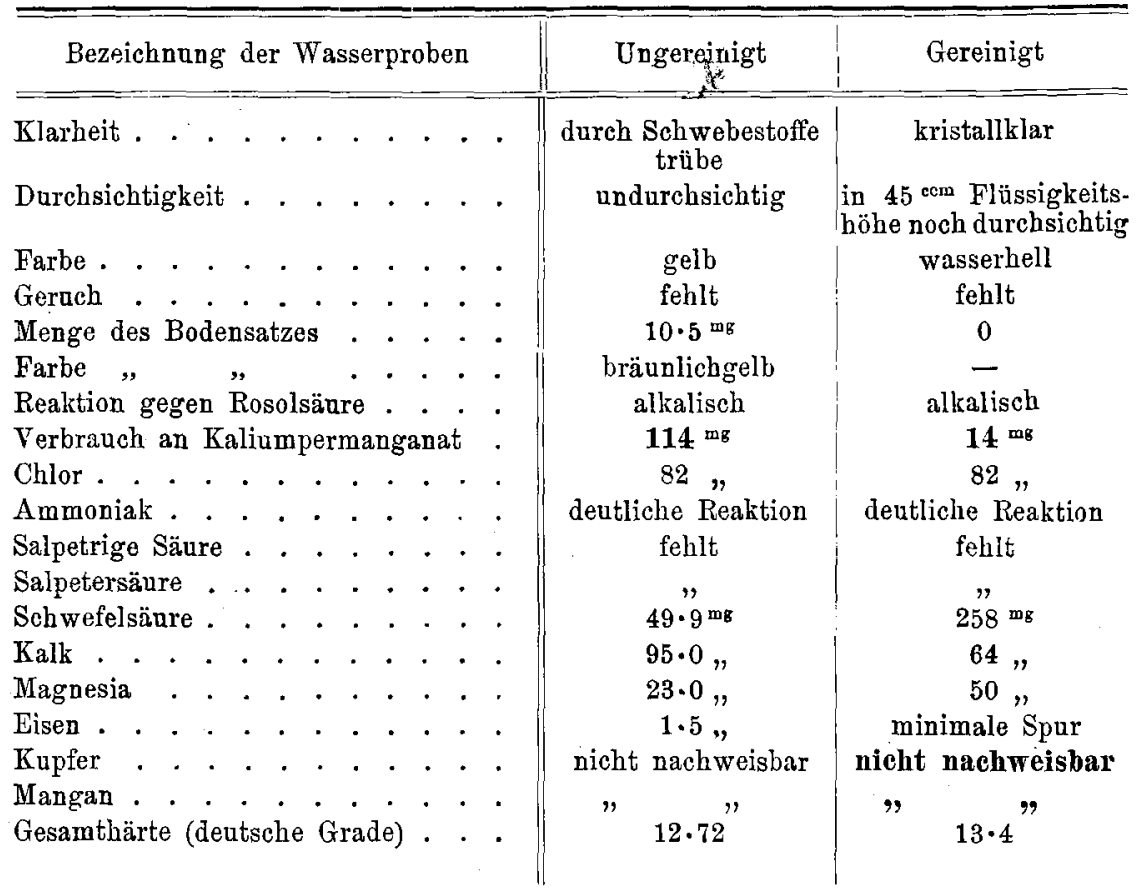


Um jede Voreingenommenheit auszuschlieBen, habe ich die Untersuchung des ungereinigten und gereinigten Wassers auch von dem chemischen und bakteriologisch-mikroskopischen Institut des Dr. Aufrecht, Berlin (NW., Albrechtstraße 11) ausführen lassen; deren wesentlichste Resultate teile ich umstehend mit.

Aus dieser Untersuchung erhellt, daB mit dem bakteriologischen Effekt eine in die Augen fallende physikalische und deutlich nachweisbare chemische Reinigung Hand in Hand geht.

Besonders fällt auf, daB der Gehalt an organischer Substanz a uf $1 / 8$ heruntergegangen ist. Die Zunahme des Abdampfrückstandes und des Glühverlustes bei dem gereinigten Wasser ist so zu erklären, daB die aus dem Kupfersulfat freiwerdende Schwefelsäure die im Rohwasser gelösten Kalkund Magnesiasalze (Karbonate und Bikarbonate) in Kalksulfat und Magnesiumsulfat umwandelt und aus dem Filtergewebe Spuren ron Magnesiasalzen in Lösung gehen, wie die Zunahme der Magnesia im gereinigten Wasser beweist. Im Zusammenhang damit erklärt sich auch die Zunahme der Gesamthärte. Das Eisen ist zu Ferriverbindungen oxydiert und ausgefallen.

Das Wichtigste an dem Untersuchungsbefund ist jedoch, daB in dem gereinigten Wasser keine Spuren von Kupfer oder Mangan nachweisbar, d. h. also alle zugesetzten gesundheitsschädlichen Substanzen wieder aus dem gereinigten Wasser verschwunden sind. Das günstige Urteil über die Geschmacklosigkeit des Wassers findet also seine Stütze in dem objektiven Untersuchungsbefund.

Wie ist nun der Desinfektionseffelt des Verfahrens zu erklären, und welche chemischen Umsetzungen bedingen das Verschwinden der Desinfizienten aus dem Filtrat, während der Filterschlamm noch bakterizide Eigenschaften aufweist?

Die Desinfektionswirkung ist meiner Utberzeugung nach eine dreifache. Einmal wirkt das Kaliumpermanganat, wie bereits erwähnt, durch Abgabe atomistischen Sauerstoffs, zweitens das Kupfersulfat in seiner bakteriziden Eigenschaft als Kupfersalz und drittens die Kombination beider Substanzen, indem das Kupfersulfat als Katalysator die Abspaltung des atomistischen Sauerstoffs aus dem Kaliumpermanganat verstärkt.

Die desinfizierende Wirkung ron Kupfersalzen ist besonders von amerikanischer Seite für Wasserversorgungsanlagen benutzt worden, und zwar nicht allein um das Algenwachstum zu verhindern, sondern auch zur Abtötung pathogener Bakterien (Moore, Kellermann, Beckwith). Clark und Gage stellten jedoch fest, daB die Desinfektionswirkung erst bei einer Konzentration ron 1:1000 zuveriässig eintritt, wodurch das Wasser natürlich zum Genuß unbrauchbar wird. Da bei meinem Verfahren diese Konzentration fast erreicht wird, so ist der Anteil des Kupfer- 
sulfats allein schon nicht zu unterschätzen. Von der Wirkung des Sauerstoffes und zwar in atomistischer Form überzeugt am besten der aus der Mischung der beiden Chemikalien in Wasser aufsteigende Geruch nach Ozon.

Bereits vor Zusatz des als Neutralisationsmittel dienenden Wasserstoffsuperoxyds tritt bei den Desinfizientien eine Umsetzung ein, welche sich in dem Auftreten eines geringen Niederschlages dokumentiert und, wie ich vorausschicke, ganz der gleichen Art ist, wie die durch das Wasserstoffsuperoxyd bedingte, welche die Chemikalien völlig zerstört. Das Kaliumpermanganat wird auf dem Umwege über das Manganosulfat zu Mangansuperoxyd (Braunstein) oxydiert, welches als unlöslicher Niederschlag ausfällt. Aus dem Kupfersulfat wird unter Freiwerden der Schwefelsäure Kupferoxyd, ein schwarzes, im Wasser unlösliches Pulver, welches fein verteilt in den Schlamm übergeht und die im Schlamm zurückgebliebenen Keime rernichtet.

Unter den gediegenen Metallen steht das Kupfer bekanntlich in bezug auf bakterizide Wirkung an erster Stelle, so daB Krämer auf Grund seiner Untersuchungen direkt rorschlägt, Wasser durch Kupfer zu sterilisieren. Mit Bouillonkulturen von Typhus- oder Cholerabakterien infiziertes und danach filtriertes Wasser wurde nach 2 bis 4 Stunden steril, wenn Kupferplatten oder Münzen von etwa 9 qom Oberfläche pro Liter Wasser hineingebracht wurden. Christian, welcher die Bedeatung der gediegenen Metallle als Desinfertionsmittel eingehender untersucht hat, stellt gleichfails fest, daß Darmkeime auf Kupfer, Messing, Zink noch niemals gefunden worden sind, wobei er die Frage offen läBt, ob die Abtötung der Keime durch gewöhnliche Giftwirkung oder auf oligodynamischem Wege zustande kommt. $\mathrm{DaB}$ die nach Zusatz des Wasserstoffsuperoxyds auftretende massenhafte Sauerstoffentwicklung auch noch einen desinfektorischen Effekt äuBert, möchte ich nach meinen Erfahrungen verneinen, da der gasförmige, molekulare Sauerstoff keine bakteriziden Eigenschaften besitzt.

Erfüllt nun dieses kombinierte chemisch-physikalische Verfahren die eingangs erwähnten an ein unter Feldverhältnissen brauchbares Wassersterilisationsverfahren zu stellenden Anforderungen?

Das Verfahren setzt den Soldaten in den Stand, sich in 15 Minuten aus stark verunreinigtem Oberflächenwasser klares, geruch- und geschmackloses Wasser herzustellen, welches frei von pathogenen Mikroorganismen und gesundheitsschädlichen Substanzen ist. Ls ist einfach und leicht anwendbar, der Filter sowie die Chemikalien sind feldmäBig zu transportieren und genügend lange Zeit haltbar, wenn sie entsprechende, aber durchaus praktisch mögliche Verpackung erhalten. Die Reinigung der Filter wird allein durch die verringerte Filterleistung bedingt, die jeder sicher be- 
urteilen kann, und besteht in dem Abbürsten der Filterkörper mit irgend welchem zur Verfügung stehenden Wasser und nachfolgendem Ausglühen an offenem Feuer. Will man diese Reinigung, was vielleicht ganz zweckmäBig wäre, nur dem Sanitätspersonal überlassen, so genügt es, den Filterkörper mit einer besonders geformten Mutter anzuschrauben, auf die nur ein besonderer Schlüssel paßt. Rechnet man als durchschnittliche Filterleistung in 4 Minuten 1 Liter und pro Mann 1/2 Liter, so könnten in $1 / 2$ Stunde 8 Mann aus einem Filter versorgt werden. Nach bereits angestellten Versuchen ist es jedoch möglich, für das Verfahren geeignete, größere Filter herzustellen, sogar solche, welche unter Pumpendruck arbeiten und, ohne die Qualität des Wassers zu beeinträchtigen, eine ganz bedeutend gröBere Filterleistung aufweisen. 


\section{Literatur-Verzeichnis.} Berlin 1911.

Christian, Die Bedeutung gediegener Metalle als Desinfektionsmittel.

Clark u. Gage, Journ. of Inf. Dis. 1906. Suppl. II. S. 175.

Croner, Diese Zeitschrift. 1909. Bd. LXIII. S. 326.

Ferrand et Lambert, Revue d'hygiène et de police sanitaire. 1908. Bd. XXX. S. 553 .

Glaser, Der Militärarat. 28. Januar 1910. S. 22.

Gottstein, Virchows Archiv. Bd. LXXXIII. Hft. 2.

Hetsch, Lehrbuch der Militärhygiene. Berlin 1910. Bd. II. S. 321.

Derselbe, Gedenkschrift für Dr. Rud. von Leuthold. 1906. Bd. I. S. 205.

Jorns, Archiv f. Hygiene. 1908. Bd. LXVII. S. 134.

Kellermann and Beckwith, Ut. Dep. of Agricult. Bureau of Plant Industry, Bull. Nr. 100. Part. 7. Washington 1906.

Krämer, American Journal of Pharmacy. 1906. Vol. LXXVIII. p. 140.

Küster, Archiv f. Hygiene. 1904. Bd. I. S. 364.

Moore and Kellermann, $\mathcal{A}$ Method of Destroying or Preventing the Growth of Algae and certain Pathogenic Bacteria in Water Supplies. Washington 1904.

Dieselben, Copper as an Algicide and Desinfectant in Water Supplies. Washington 1905.

Raudnitz, Ergebnisse der Plysiologie. 1903. Bd. II.

Reichel, Diese Zeitschrift. 1908. Bd. LXI. Hft. 8.

Schmidt, Centralblatt für Baleteriologie und Parasitenkunde. 1910. Abt. I. Bd. LV. Orig. S. 327.

Schüder, Diese Zeitschrift. 1901. Bd. XXXVII. S. 307.

Derselbe, Ebenda. 1902. Bd. XXXIX. S. 532.

Derselbe, Ebenda. 1902. Bd. XL. S. 196.

Schumburg, Deutsche med. Wochenschrift. 1897. S. 145 u. $407 \mathrm{ff}$.

Trangott, Diese Zeitschrift. 1893. Bd. XIV. S. 440. 